\title{
Significance of Post-Alcohol Septal Ablation Right Bundle Branch Block
}

\author{
Yuichiro Maekawa, MD, PhD
}

A lcohol septal ablation (ASA) reduces the extent of left ventricular outflow tract obstruction, mitral regurgitation due to systolic anterior motion, and left ventricular diastolic dysfunction, thus improving heart failure symptoms in drug-refractory hypertrophic obstructive cardiomyopathy (HOCM) patients.

In a 1995 issue of The Lancet, Ulrich Sigwart introduced ASA, as performed on 3 cases. ${ }^{1}$ Since then, ASA has become an alternative for patients with HOCM that is drug-resistant and for whom myectomy is not indicated due to advanced age or high risk of open-heart surgery. In 1998, Faber et al published a report in Circulation concerning selective septal ablation using myocardial contrast echocardiography, a technique that has subsequently improved clinical outcomes and reduced complications. ${ }^{2}$ In the era of contemporary ASA, preprocedural cardiac computed tomography may also be useful for improving clinical outcomes and reducing complications. ${ }^{3}$

The complications of ASA include ventricular tachycardia/fibrillation and bradyarrhythmia such as complete atrioventricular (AV) block, left anterior descending artery (LAD) occlusion due to ethanol leakage, access site-related vascular complications, and cardiac tamponade associated with temporary pacemaker insertion and placement. ${ }^{4,5} \mathrm{Of}$ these, complete AV block is the most frequent complication., ${ }^{46}$ The frequency of complete AV block, including temporary cases, has remained high at $10-40 \%$, though the slow injection technique can help lower the rate. ${ }^{7}$ ASA confers changes in AV and intraventricular conduction. In general, 1st-degree AV block, right bundle branch block (RBBB), left anterior fascicular block (LAFB), left posterior fascicular block, and left bundle branch block (LBBB) have been reported after ASA (Table). The ECG changes during and after ASA has been extensively reported, ${ }^{7-9}$ and according to those reports, RBBB occurs more frequently than complete AV block, has no negative effects on hemodynamics, and is not considered an ASA-related complication. A report from the Euro-ASA registry revealed that development of BBB, including RBBB and LBBB, after ASA was not associated with poor clinical outcomes.10 However, the significance of RBBB after ASA remains uncertain in the Japanese population.

In this issue of the Journal, Matsuda et a ${ }^{11}$ retrospectively analyze the importance of RBBB $24 \mathrm{~h}$ after the ASA

\section{Article p 1481}

procedure in the largest Japanese ASA cohort and report that they found no apparent negative effects on clinical prognosis after at least 1 year. This has been recognized by ASA-experienced operators, but clear evidence based on a Japanese cohort was needed to support this. A previous study reported that the proximal LAD septal branches perfused the right bundle branch and the anterior fascicle of the left bundle branch in $90 \%$ of cases, whereas the right coronary artery (via the atrioventricular-nodal artery) perfused the posterior fascicle of the left bundle branch in $90 \%$ of cases. ${ }^{12}$ This finding indicated that proximal LAD occlusions can cause RBBB and/or LAFB. Therefore, it is easily understandable that a higher rate of RBBB occurs during or/and after ASA. ${ }^{8}$

Previous studies, ${ }^{6,13,14}$ and the report by Matsuda et a ${ }^{11}$ have demonstrated the relationship between the volume of ethanol and the occurrence of complete AV block or BBB. The volume of ethanol is also associated with peak creatine kinase (CK) levels, which indicate the infarct size after ASA. ${ }^{15}$ Therefore, the occurrence of complete AV block or BBB may relate to the infarct size after ASA. In addition, patients with CK levels $<1,300$ IU/L after ASA have demonstrated worse clinical outcomes than those with higher CK levels. ${ }^{15}$ Taken together, these findings suggest that the

\begin{tabular}{|lc|}
\hline \multicolumn{2}{|c|}{$\begin{array}{c}\text { Table. Cardiac Conduction Disturbance After Alcohol Septal } \\
\text { Ablation }\end{array}$} \\
Cardiac conduction disturbance & Frequency \\
1st-degree AV block & Rarely \\
RBBB & Often \\
LAFB & Rarely \\
LPFB & Almost never \\
RBBB+LAFB & Rarely \\
RBBB+LPFB & Almost never \\
Complete AV block & Occasionally \\
\hline
\end{tabular}

Frequency: transient and permanent conduction disturbance. $\mathrm{AV}$, atrioventricular; LAFB, left anterior fascicular block; LPFB, left posterior fascicular block; RBBB, right bundle branch block.

The opinions expressed in this article are not necessarily those of the editors or of the Japanese Circulation Society.

Received April 19, 2021; accepted April 20, 2021; J-STAGE Advance Publication released online June 3, 2021

Division of Cardiology, Internal Medicine III, Hamamatsu University School of Medicine, Hamamatsu, Japan

Mailing address: Yuichiro Maekawa, MD, PhD, Division of Cardiology, Internal Medicine III, Hamamatsu University School of

Medicine, 1-20-1 Handayama, Higashi-ku, Hamamatsu 431-3192, Japan. E-mail: ymaekawa@hama-med.ac.jp

All rights are reserved to the Japanese Circulation Society. For permissions, please e-mail: cj@j-circ.or.jp

ISSN-1346-9843 
long-term prognosis, particularly the long-term alleviation of heart failure symptoms, is not necessarily worse in patients with complete AV block or BBB after ASA, though complete AV block especially is a serious cardiac conduction disturbance and is often thought to have a negative effect on clinical outcomes. ${ }^{6,14}$ In fact, higher peak CK levels and a larger number of injected septal arteries were observed in the RBBB group compared with the no$\mathrm{RBBB}$ group, and the frequency of repeat ASA was significantly lower in the RBBB group than in the no-RBBB group in Matsuda et al's report. ${ }^{11}$ However, due to the phenotypic diversity in HOCM, the effectiveness of ASA cannot be predicted only by infarct size and/or peak CK level. It should be confirmed by multiple evaluation methods of the efficacy of ASA in relation to long-term clinical outcomes. ${ }^{14}$ In addition, it is still controversial whether patients should receive low or high doses of ethanol to improve long-term clinical outcomes, because patients with drug refractory HOCM are diverse. However, there may be a trade-off between long-term symptomatic improvement with extensive myocardial ablation and the occurrence of complete AV block during and/or after ASA.

The report by Matsuda et $\mathrm{al}^{\mathbf{1 1}}$ should be interpreted with caution for the following reasons: (1) subjects with LBBB were included in the no-RBBB group, and (2) subjects with RBBB showing transient complete AV block were included in the RBBB group. These ECG changes may affect the clinical outcomes. Although not emphasized in the report, ASA operators should be aware of the fact that those subjects who underwent ASA procedures should have exhibited higher frequencies of RBBB, when ASA operators performed the procedure in patients exhibiting LBBB in their baseline ECG. This is because a complete AV block will definitely occur during and/or after the procedure. In such patients, DDD pacemaker implantation should be considered beforehand. Although the post-ASA ECG changes are an old topic, they emphasize clinically essential issues and therefore, may be an essential reference for those involved with ASA procedures.

\section{Disclosures}

Y.M. received Donations Scholarship funds from Abbott Medical Japan LLC, Terumo Corporation, Medtronic Japan Co., Ltd, and Biotronik Japan Co., Ltd.

\section{References}

1. Sigwart U. Non-surgical myocardial reduction for hypertrophic obstructive cardiomyopathy. Lancet 1995; 346: 211-214.

2. Faber L, Seggewiss H, Gleichmann U. Percutaneous transluminal septal myocardial ablation in hypertrophic obstructive cardiomyopathy: Results with respect to intraprocedural myocardial contrast echocardiography. Circulation 1998; 98: 2415-2421.

3. Maekawa Y, Akita K, Takanashi S. Contemporary septal reduction therapy in drug-refractory hypertrophic obstructive cardiomyopathy. Circ J 2018; 82: 1977-1984.

4. Sorajja P, Ommen SR, Holmes DR Jr, Dearani JA, Rihal CS, Gersh BJ, et al. Survival after alcohol septal ablation for obstructive hypertrophic cardiomyopathy. Circulation 2012; 126: $2374-$ 2380.

5. Rigopoulos AG, Seggewiss H. A decade of percutaneous septal ablation in hypertrophic cardiomyopathy. Circ $J$ 2011; 75: $28-$ 37.

6. Veselka J, Jensen MK, Liebregts M, Januska J, Krejci J, Bartel $\mathrm{T}$, et al. Long-term clinical outcome after alcohol septal ablation for obstructive hypertrophic cardiomyopathy: Results from the Euro-ASA registry. Eur Heart J 2016; 37: 1517-1523.

7. Chang SM, Nagueh SF, Spencer WH 3rd, Lakkis NM. Complete heart block: Determinants and clinical impact in patients with hypertrophic obstructive cardiomyopathy undergoing nonsurgical septal reduction therapy. J Am Coll Cardiol 2003; 42: 296-300.

8. Runquist LH, Nielsen CD, Killip D, Gazes P, Spencer WH 3rd. Electrocardiographic findings after alcohol septal ablation therapy for obstructive hypertrophic cardiomyopathy. Am J Cardiol 2002; 90: $1020-1022$.

9. Holmes DR Jr, Valeti US, Nishimura RA. Alcohol septal ablation for hypertrophic cardiomyopathy: Indications and technique. Catheter Cardiovasc Interv 2005; 66: 375-389.

10. Jensen M, Faber L, Liebregts M, Januska J, Krejci J, Bartel T, et al. Effect of impaired cardiac conduction after alcohol septal ablation on clinical outcomes: Insights from the Euro-ASA registry. Eur Heart J Qual Care Clin Outcomes 2019; 5: 252258.

11. Matsuda J, Takano H, Morooka M, Imori Y, Nakata J, Kitamura $\mathrm{M}$, et al. Relationship between procedural right bundle branch block and 1-year outcome after alcohol septal ablation for hypertrophic obstructive cardiomyopathy: A retrospective study. Circ J 2021; 85: 1481-1491.

12. Frink RJ, James TN. Normal blood supply to the human His bundle and proximal bundle branches. Circulation 1973; 47: $8-18$

13. Veselka J, Lawrenz T, Stellbrink C, Zemanek D, Branny M, Januska J, et al. Early outcomes of alcohol septal ablation for hypertrophic obstructive cardiomyopathy: A European multicenter and multinational study. Catheter Cardiovasc Interv 2014; 84: $101-107$.

14. Akita K, Tsuruta H, Yuasa S, Murata M, Fukuda K, Maekawa Y. Prognostic significance of repeated brain natriuretic peptide measurements after percutaneous transluminal septal myocardial ablation in patients with drug-refractory hypertrophic obstructive cardiomyopathy. Open Heart 2018; 5: e000786.

15. Chang SM, Lakkis NM, Franklin J, Spencer WH 3rd, Nagueh SF. Predictors of outcome after alcohol septal ablation therapy in patients with hypertrophic obstructive cardiomyopathy. Circulation 2004; 109: 824-827. 